\title{
Segmented Cantilever and Array Configurations for Wider Frequency Band and Higher Power Generation in Piezoelectric Vibration Energy Harvester
}

\author{
Aicheng Zou $\mathbb{D}^{1,2}$ Jiefeng Li, ${ }^{1}$ Xingguo Han, ${ }^{2}$ and Qunying Wang ${ }^{2}$ \\ ${ }^{1}$ State Key Laboratory of Mechanics and Control of Mechanical Structures, Nanjing University of Aeronautics and Astronautics, \\ Nanjing 210016, China \\ ${ }^{2}$ College of Mechanical Engineering, Guilin University of Aerospace Technology, Guilin 541004, China
}

Correspondence should be addressed to Aicheng Zou; zouaicheng@guat.edu.cn

Received 9 April 2021; Revised 15 May 2021; Accepted 15 July 2021; Published 10 August 2021

Academic Editor: Wenqing Wu

Copyright (C) 2021 Aicheng Zou et al. This is an open access article distributed under the Creative Commons Attribution License, which permits unrestricted use, distribution, and reproduction in any medium, provided the original work is properly cited.

\begin{abstract}
This letter reports a piezoelectric vibration energy harvester which energy conversion efficiency is significantly improved by arraying piezoelectric sheets on cantilever beams, and the operation frequency band is widened by applying two-segment cantilever beams. A prototype is developed and tested. In this case, two group piezoelectric arrays are combined on the cantilever beams with the optimum load resistance. The total output power remains above $6.54 \mathrm{~mW}$ within the operation frequency band ranges from $27.5 \mathrm{~Hz}$ to $37.5 \mathrm{~Hz}$ when the generator is under an acceleration of $0.7 \mathrm{~g}$ and reaches two power peaks: $20.5 \mathrm{~mW}$ at $29.2 \mathrm{~Hz}$ and $12.95 \mathrm{~mW}$ at $35.4 \mathrm{~Hz}$.
\end{abstract}

\section{Introduction}

With the rapid development of the applications of wireless sensor networks, particular in some low-power conditions, wireless sensors with the function of converting the energy in the environment into electric energy are much desired. In recent years, vibration energy harvester (VEH) based on piezoelectric has attracted extensive attention of researchers [1-4]. Traditional VEHs are designed to work only in resonant mode so that they must be designed for specific environments, which results in narrow frequency band since environmental vibration is usually broadband and timevarying. In addition, the performance of VEHs declines drastically when the ambient vibration frequency deviates from their resonant frequency which poses a big challenge for energy collection [5]. Therefore, VEHs that with high efficiency of energy conversion and that can respond to lowfrequency vibrations with a time-variant frequency broadband operation range are considered as the very promising solutions [6].

The contributions of this manuscript are as follows: (i) Designing a two-segment cantilever beam piezoelectric vibration energy harvester $(\mathrm{C}-\mathrm{VEH})$ based on array arrangement of piezoelectric patches on the beam for improving the energy harvesting efficiency and working frequency bandwidth of the piezoelectric energy harvesting structure

(ii) Establishing the electromechanical conversion analysis model and its control equation under the influence of load coupling of piezoelectric composite cantilever vibration energy harvester and deducting the mathematical models of its output voltage and output power for analyzing the performance of piezoelectric vibration energy acquisition structure accurately

(iii) Analyzing the energy harvesting performance of CVEH experimentally and compared with the traditional nonarray piezoelectric cantilever vibration energy harvester (T-VEH)

The rest of the manuscript is organized as follows: In Section 2, related works are discussed followed by the proposed 
two-segment cantilever beam piezoelectric vibration energy harvester based on array arrangement of piezoelectric patches on the beam explanation in Section 3. Section 4 discusses the electromechanical conversion analysis model of piezoelectric composite cantilever energy harvester and its control equation with load coupling and the mathematical models of its output voltage and output power. Section 5 simulates and analyzes the C-VEH. Section 6 discusses the experimental analysis and summarizes a comparative assessment, and the manuscript is concluded in Section 6.4.

\section{Related Works}

Researchers have proposed several methods to improve the energy conversion efficiency and expand the band of the operation frequency of VEHs. One of the approaches is frequency tuning, such as mechanical tuning [7-11], circuit tuning, and magnetic tuning [12-15]. This strategy allows VEHs to adjust their resonant frequency according to the environmental vibration frequency. However, this would increase the complexity or consume extra energy of the VEHs. For instance, Eichhorn et al. [7] proposed a piezoelectric energy harvesting structure of cantilever beam with additional mechanical mass, which changes the resonant frequency of the structure by applying pressure or tensile force to the cantilever beam. Fan et al. [15] designed a vibration energy harvester composed of piezoelectric, stopper, and magnet. The system can tune the vibration energy harvester to a lower working frequency range by changing the gap between mass and magnets, so as to improve the efficiency of energy collection. Another approach is to use multimodal energy capture technology that has different resonant frequencies, including generator arrays [16-19] and coupled vibration [20-25]. Although coupled vibration is easy to achieve, the maximum output power of the $\mathrm{VEH}$ would be consequently reduced. Nabavi and Zhang [16] proposed a piezoelectric MEMS harvester with symmetrical and double clamping structure, which has multimode and nonlinear characteristics and effectively broadens the working bandwidth. $\mathrm{Hu}$ and $\mathrm{Xu}$ [20] proposed a broadband multimass and multispring piezoelectric vibration energy harvester based on folded asymmetric gap cantilever beam, which can make each stage of pure bending form multiple resonance modes. The working frequency band of the structure is widened effectively. Recently, nonlinear vibration energy harvesting approaches, for instance, the nonlinear magnetic coupling [26-32] and piecewise-linear structure [33-36], have attracted the interest of investigators. Although energy conversion efficiency of the unit volume material is decreased, operation frequency band of the $\mathrm{VEH}$ could be widened. The new piezoelectric structure which can broaden the frequency band and increase the efficiency of energy capture is the aim that researchers have been exploring, and it attracts a lot of researchers.

\section{Description of the C-VEH Model Design}

This letter reports a high-efficiency and broadband vibration energy harvester based on the structure of two-segment can- tilever beams with piezoelectric array configuration structure (C-VEH), as shown in Figure 1. Figure 1(a) is the model of the $\mathrm{C}-\mathrm{VEH}$, and Figure 1(b) is the proposed $\mathrm{C}-\mathrm{VEH}$ design which includes the side view and top view of the structure. The proposed $\mathrm{C}-\mathrm{VEH}$ consists of primary cantilever beam (the $1^{\text {st }}$ ), secondary cantilever beam (the $2^{\text {nd }}$ ), and two suspended masses M1 and M2. The left end of the $1^{\text {st }}$ is fixed to the base of the structure, and the mass M1 is suspended from the right end of the $1^{\text {st }}$. The right end of the $2^{\text {nd }}$ is fixed to $\mathrm{M} 1$, and the mass $\mathrm{M} 2$ is then suspended to the left end of the $2^{\text {nd }}$. There are two piezoelectric sheets of the same size attached on the upper surface of each cantilever beam. The base of the structure is fixed on the excitation platform.

The operation frequency band of the C-VEH is widened by applying two-segment cantilever beams. The structure of two-segment cantilever beams has two close resonant frequencies, which responds to ambient low-frequency vibration. To improve the energy capture efficiency, the piezoelectric arrays isolated by gaps are installed on the cantilever beams and electrically independent from each other.

\section{Theoretical Analysis}

4.1. The Governing Equation. For the vibration energy harvester of piezoelectric composite cantilever beam (VEH) shown in Figure 2, the traditional method is to use lumped parameter for modeling. The system is equivalent to the lumped parameter model composed of mass, stiffness, damping, and piezoelectric (as shown in Figure 3), in which the mass $(M)$ is on the spring with stiffness $K$, the damper with coefficient $(\eta)$, and the piezoelectric element with piezoelectric coefficient $\Theta$, the electric field is along the thickness direction of the piezoelectric layer, and the piezoelectric layer works in $\mathrm{d}_{31}$ mode.

The main limitation of the model is that it assumes the motion characteristics of the piezoelectric cantilever are independent of the load, that is, it belongs to the state of no-load coupling. But the actual situation is due to the physical characteristics of the piezoelectric structure; system vibration leads to the electricity generated by the piezoelectric layer, and after the load consumption, it will have an impact on the stiffness of the piezoelectric layer itself, which is reflected by the piezoelectric circuit coupling.

Therefore, considering the load coupling effect, the traditional lumped parameter model is improved to predict the dynamic characteristics of VEH more accurately. The equivalent model of the system is shown in Figure 4. The lumped parameters in the equivalent model include equivalent mass $\left(M_{\text {eq }}\right)$, equivalent stiffness $\left(K_{\mathrm{eq}}\right)$, system damping $(\eta)$, equivalent current source $(\Theta \dot{z}(t))$, and equivalent capacitance $\left(C_{\mathrm{eq}}\right.$ ) (the upper and lower piezoelectric parts are connected in series), the external circuit includes rectifier bridge, rectifier capacitor $\left(C_{o}\right)$, and load $(R)$. The piezoelectric part is the link between the load part and the vibration part, which is equivalent to two parts equivalent current source $(\Theta \dot{z}(t))$ and equivalent capacitance $\left(C_{\mathrm{eq}}\right)$.

The control equation of the equivalent model of $\mathrm{VEH}$ under the influence of load coupling shown in Figure 4 is as 

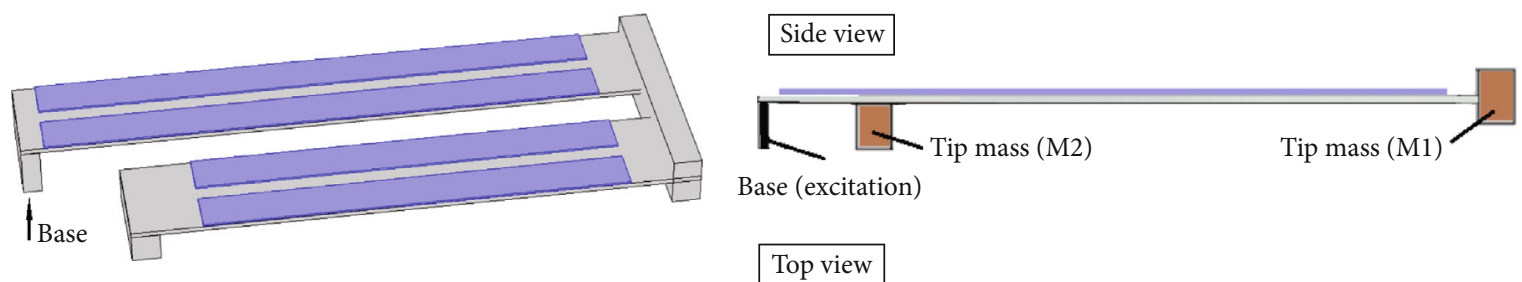

Base (excitation)

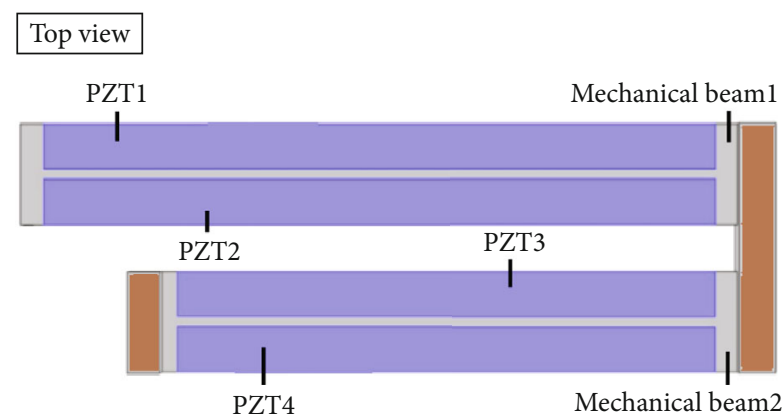

(a)

(b)

Figure 1: Structure of C-VEH: (a) mode of C-VEH; (b) the proposed C-VEH design.

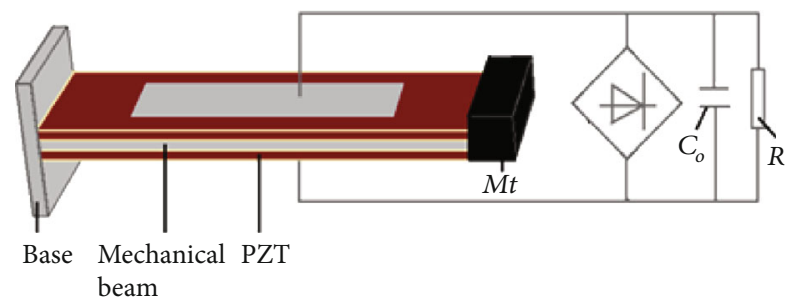

Figure 2: Structure diagram of energy harvester of piezoelectric composite cantilever.

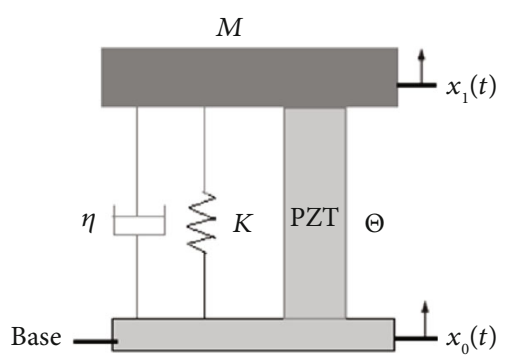

Figure 3: The traditional lumped parameter mode.

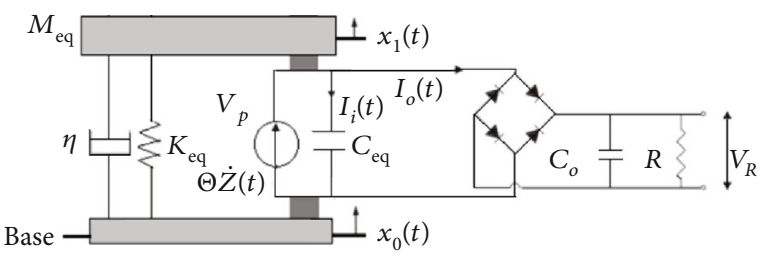

FIGURE 4: VEH equivalent analysis model with load coupling influence. follows [37]:

$$
\begin{gathered}
M_{\mathrm{eq}} * \ddot{x}_{1}(t)+\eta *\left[\dot{x}_{1}(t)-\dot{x}_{0}(t)\right]+K_{\mathrm{eq}} *\left[x_{1}(t)-x_{0}(t)\right]=0, \\
\Theta * \dot{z}(t)=I_{i}(t)+I_{o}(t),
\end{gathered}
$$

where

$$
\begin{gathered}
z(t)=x_{1}(t)-x_{0}(t), \\
I_{i}(t)=C_{\mathrm{eq}} * \dot{v}_{P}(t), \\
I_{o}(t)=\frac{V_{R}}{R}+C_{o} * v_{R}(t) .
\end{gathered}
$$

Equations (1) and (2) are the governing equation of the system considering the influence of load coupling, and $z(t)$ is the displacement of the cantilever beam relative to the base. Combined with the motion boundary conditions of the system, the vibration displacement, output voltage, and output power of VEH can be further solved.

4.2. Output Voltage and Vibration Displacement Response. The excitation source of the system is a sine function, and the vibration displacement response of the cantilever beam can also be regarded as a sine function. It is obvious that $z$ ( $t), v_{P}(t)$, and $v_{R}(t)$ in equation (2) are also sine functions. By integrating the two sides of equation (2), we can get the following:

$$
\int_{a}^{b} \Theta * \dot{z}(t) d t=\int_{a}^{b} C_{\mathrm{eq}} * \dot{v}_{P}(t) d t+\int_{a}^{b} C_{o} * v_{R}(t) d t+\int_{a}^{b} \frac{V_{R}}{R} d t
$$

According to the method, [37] can solve equation (4), that is, let $b-a=\mathrm{T} / 2$ ( $T$ is the period of sine function) in equation (4), and $z(t), v_{P}(t)$, and $v_{R}(t)$ be the minimum 
and maximum at the points $a$ and $b$, respectively. Let $z(t)$ $=Z_{0} \sin \omega t, v_{P}(t)=V_{P} \sin \omega t$, and $v_{R}=V_{R} \sin \omega t$, and after the system is stable, $\int_{a}^{b} C_{\text {eq }} * \dot{v}_{P}(t) d t=0, V_{P}=V_{R}$. From equation (2), it can be concluded that

$$
2 \Theta Z_{0}=2 C_{\text {eq }} V_{R}+\frac{T}{2} \frac{V_{R}}{R} .
$$

Since $\omega=2 \pi / T$, so

$$
V_{R}=\frac{R \omega \Theta}{C_{\mathrm{eq}} \omega R+\pi / 2} Z_{0}
$$

Equation (6) reflects the relationship between the amplitude of vibration displacement response and the amplitude of output voltage. By solving $Z_{0}, V_{R}$ can be obtained, and then, the power of the load can be obtained.

4.3. Amplitude of Vibration Displacement Response. Let $x_{0}(t$ )$=X_{0} e^{j \omega t}, x_{1}(t)=X_{1} e^{j \omega t}$, and $z(t)=Z_{0} e^{j \omega t}=X_{1} e^{j \omega t}-X_{0} e^{j \omega t}$, where $X_{0}, X_{1}$, and $Z_{0}$ are the amplitudes of the response of the excitation source and the cantilever beam, respectively. By substituting them into equation (1), we can get the following results:

$M_{\mathrm{eq}} *\left(-\omega^{2} X_{0} e^{j \omega t}\right)+\eta *\left[j \omega X_{1} e^{j \omega t}-j \omega X_{0} e^{j \omega t}\right]+K_{\mathrm{eq}} *\left[X_{1} e^{j \omega t}-X_{0} e^{j \omega t}\right]=0$.

By solving equation (7), the following results can be obtained:

$$
Z_{0}=\frac{\omega^{2} M_{\mathrm{eq}}}{j \omega \eta+K_{\mathrm{eq}}-\omega^{2} M_{\mathrm{eq}}} X_{0 .}
$$

In equation (8), $X_{0}$ and $Z_{0}$ are the amplitude of vibration displacement response of excitation source and cantilever beam, respectively. Let

$$
\omega=\sqrt{\frac{k_{\mathrm{eq}}}{M e \mathrm{q}}}, \xi=\frac{\eta}{2 \sqrt{K_{\mathrm{eq}} M_{\mathrm{eq}}}}, \Delta=\frac{\omega}{\omega_{n}} .
$$

By substituting (9) into equation (8), we can get the following results:

$$
Z_{0}=\frac{\Delta^{2}}{1-\Delta^{2}+2 j \xi \Delta} X_{0}
$$

4.4. Output Voltage and Power. Substituting equation (10) into equation (6), the output voltage of the load can be obtained. That is,

$$
V_{R}=\frac{R \omega \Theta}{C_{\mathrm{eq}} \omega R+\pi / 2} \times \frac{\Delta^{2}}{1-\Delta^{2}+2 j \xi \Delta} X_{0}
$$

TABLE 1: The parameters of VEH.

\begin{tabular}{lcc}
\hline Object & Parameters & Value \\
\hline \multirow{3}{*}{ Elastic layer } & Material & Aluminum \\
& $l \times b \times t_{m}(\mathrm{~mm})$ & $80 \times 20 \times 0.5$ \\
& $\rho_{p}\left(\mathrm{~kg} / \mathrm{m}^{3}\right)$ & 2700 \\
& $E_{m}(\mathrm{GPa})$ & 70 \\
& Material & PZT-51 \\
& $l \times b \times t_{m}(\mathrm{~mm})$ & $80 \times 20 \times 0.2$ \\
Piezoelectric layer & $\rho_{m}\left(\mathrm{~kg} / \mathrm{m}^{3}\right)$ & 7500 \\
& $E_{P}(\mathrm{GPa})$ & 56 \\
& $\varepsilon_{33}^{s}\left(10^{-8} \mathrm{~F} / \mathrm{m}\right)$ & 3.01 \\
& $e_{31}\left(10^{-3} \mathrm{~N} / \mathrm{Vm}\right)$ & 8.4 \\
\hline
\end{tabular}

The output power of the system is as follows::

$$
\begin{aligned}
P= & \frac{V_{R}^{2}}{R}=\left[\left[R \omega \Theta /\left(C_{\mathrm{eq}} \omega R+\frac{\pi}{2}\right)\right]\right. \\
& \left.\times\left[X_{0} \Delta^{2} /\left(1-\Delta^{2}+2 j \xi \Delta\right)\right]\right]^{2} / R \\
= & R \times\left[\frac{\Delta \omega_{n} \Theta}{C_{\mathrm{eq}} \Delta \omega_{n} R+\frac{\pi}{2}} \times \frac{\Delta^{2}}{1-\Delta^{2}+2 j \xi \Delta} X_{0}\right]^{2} .
\end{aligned}
$$

Equation (12) is the amplitude expression of the output power of the system with the influence of load coupling and simple harmonic vibration excitation, which reflects the power generation capacity of $\mathrm{VEH}$ and can be used as the basis for the structural optimization design of the system. It can be seen that $P$ is not only related to the geometry and material parameters of the system but also related to the external environmental conditions (frequency $\omega$ and amplitude $X_{0}$ of the excitation signal, frequency ratio $\Delta$ (ratio of the excitation frequency to the first natural frequency of the system), and load $R$ ), in which the frequency ratio is $\Delta=\omega /$ $\omega_{n}$. Therefore, when the system structure size and the amplitude of the excitation signal are determined, the power generation of the piezoelectric composite cantilever vibration energy harvester is essentially related to the $\omega$ and $R$. Equation (12) can be rewritten as follows:

$$
P=f(\omega, R)
$$

According to equation (13), the optimal matching resistance of the system can be obtained when other structural factors and the frequency of the excitation signal are determined, that is, the $R^{\text {opt }}$ obtained by solving equation (14). The optimal excitation frequency of the system can also be obtained when the load is determined, that is, get $\omega^{\text {opt }}$ obtained by solving equation (15).

$$
\begin{gathered}
\left.\frac{\partial}{\partial R} f(\omega, R)\right|_{\omega}=0, \\
\left.\frac{\partial}{\partial \omega} f(\omega, R)\right|_{R}=0 .
\end{gathered}
$$




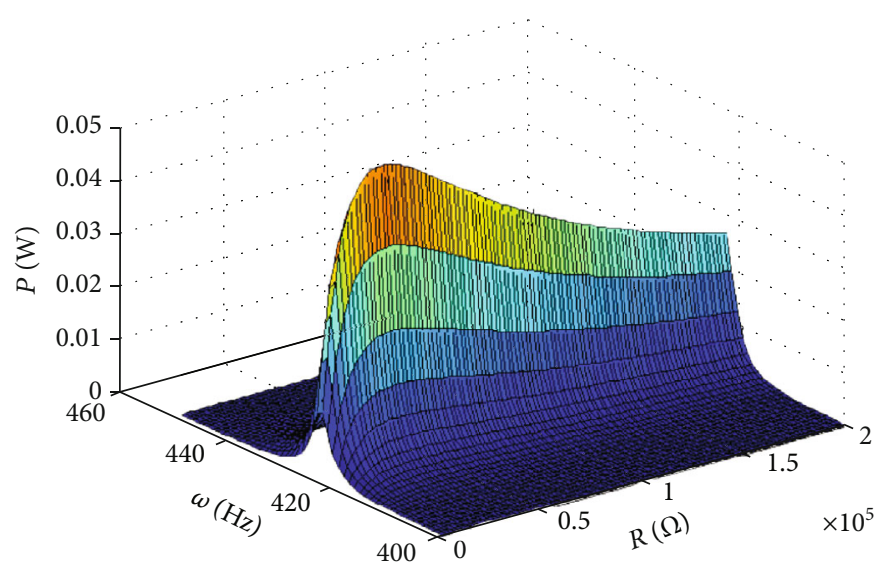

Figure 5: $P-(\omega, R)$.

TABLE 2: Parameters of the C-VEH Investigated in this letter.

\begin{tabular}{|c|c|c|c|}
\hline & & Primary cantilever beam & Secondary cantilever beam \\
\hline \multirow{4}{*}{ Piezoelectric layer (PZT) } & Dimension $(l \times w \times t)(\mathrm{mm})$ & $60 \times 5 \times 0.2(\mathrm{PZT} 1, \mathrm{PZT} 2)$ & $45 \times 5 \times 0.2(\mathrm{PZT} 3, \mathrm{PZT} 4)$ \\
\hline & Material & \multicolumn{2}{|c|}{ Ceramic PZT-5H (Lead zirconate titanate) } \\
\hline & Density $\left(10^{3} \mathrm{~kg} / \mathrm{m}^{3}\right)$ & \multicolumn{2}{|c|}{7.5} \\
\hline & Young's modulus (GPa) & \multicolumn{2}{|c|}{66} \\
\hline \multirow{4}{*}{ Mechanical beam } & Dimension $(l \times w \times t)(\mathrm{mm})$ & $70 \times 11 \times 0.6$ & $60 \times 11 \times 0.6$ \\
\hline & Material & \multicolumn{2}{|c|}{ Aluminum } \\
\hline & Density $\left(10^{3} \mathrm{~kg} / \mathrm{m}^{3}\right)$ & \multicolumn{2}{|c|}{2.7} \\
\hline & Young's modulus (GPa) & \multicolumn{2}{|c|}{60} \\
\hline \multirow{2}{*}{ Proof mass } & Material & \multicolumn{2}{|c|}{$\mathrm{Fe}$ (iron) } \\
\hline & Weight $(g)$ & $5.06(\mathrm{M} 1)$ & $2.89(\mathrm{M} 2)$ \\
\hline
\end{tabular}

4.5. Numerical Calculation. A vibration energy harvesting structure (VEH) is constructed, in which the middle is an elastic layer, and the upper and lower surfaces of the elastic layer are covered with piezoelectric layers. The parameters of $\mathrm{VEH}$ are shown in Table 1.

The first-order natural frequency of the system is estimated to be about $420 \mathrm{~Hz}$. The optimal load $R^{\mathrm{opt}}=0.28 \times$ $10^{5} \Omega$ can be calculated from equation (14). Therefore, according to equation (13), the influence of frequency $(\omega)$ and load $(R)$ of excitation signal on VEH output power is numerically analyzed by MATLAB. The frequency scanning range of excitation signal is $400 \sim 460 \mathrm{~Hz}$, and the scanning range of load is $0 \sim 2 \times 10^{5} \Omega$. The results are shown in Figure 5, and it can be seen that when $R$ is fixed, $P$ first increases and then decreases with the increase of $\omega$. When $\omega^{\mathrm{opt}}=\omega_{n}$, the output power reaches the peak. When $\omega$ is fixed, $P$ first increases and then decreases with the increase of $R$, and there is an optimal load $R^{\text {opt }}=0.28 \times 105 \Omega$ to $\max -$ imize the output power.

\section{Finite Element Simulation Analysis}

A C-VEH is developed using the aluminum sheet and the Lead zirconate titanate $(\mathrm{PZT}-5 \mathrm{H})$. The resonant frequencies of the proposed $\mathrm{C}-\mathrm{VEH}$ are closely related to the length, thickness, and weight of the cantilever beams, which are around $30 \mathrm{~Hz}$, and the band of the resonant frequencies is about $6 \mathrm{~Hz}$. The length of primary cantilever beam $(l=70$ $\mathrm{mm})$ is selected in accordance with the specific application situation of the structure, and the dimensions of the other parts of the $\mathrm{C}-\mathrm{VEH}$ are designed to achieve the requirements of resonant frequencies and band, respectively, as shown in Table 2.

The vibration modes and the resonant frequencies of the $\mathrm{C}-\mathrm{VEH}$ are analyzed via finite element simulation (by COMSOL Multiphysics ${ }^{\circledR}$ ). The material and size of piezoelectric structure are shown in Table 2. The setting of the COMSOL software is as follows: damping ratio is 0.01 and scanning frequency range is $20 \mathrm{~Hz}-40 \mathrm{~Hz}$. The results of the first two resonant modes shown in Figure 6(a) demonstrate the displacement of the beams, two peaks appear at the first two open circuit resonant frequencies $(28.01 \mathrm{~Hz}$ and $34.02 \mathrm{~Hz}$, respectively) of $\mathrm{C}-\mathrm{VEH}$, and operating frequency bandwidth of which is $6.01 \mathrm{~Hz}$. In the first resonant mode (Figure 6(b)), the deformations of the free ends of the $1^{\text {st }}$ beam and the $2^{\text {nd }}$ beam are significant, and the largest stresses occurs at the left end of the $1^{\text {st }}$ beam and right end of the $2^{\text {nd }}$ beam. Figure $6(\mathrm{c})$ shows that the left end of the $2^{\text {nd }}$ beam has a significant deformation while the $1^{\text {st }}$ beam has deformed in the second mode as well. Also, noticed that 


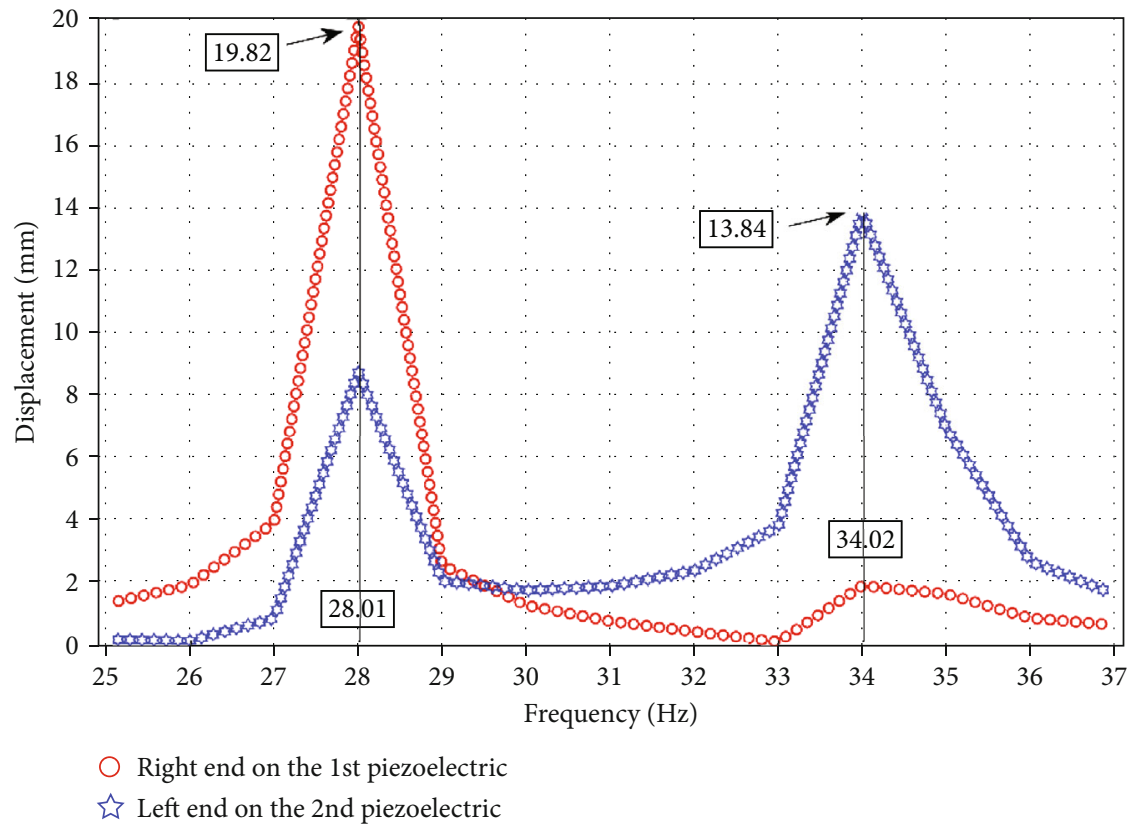

(a)

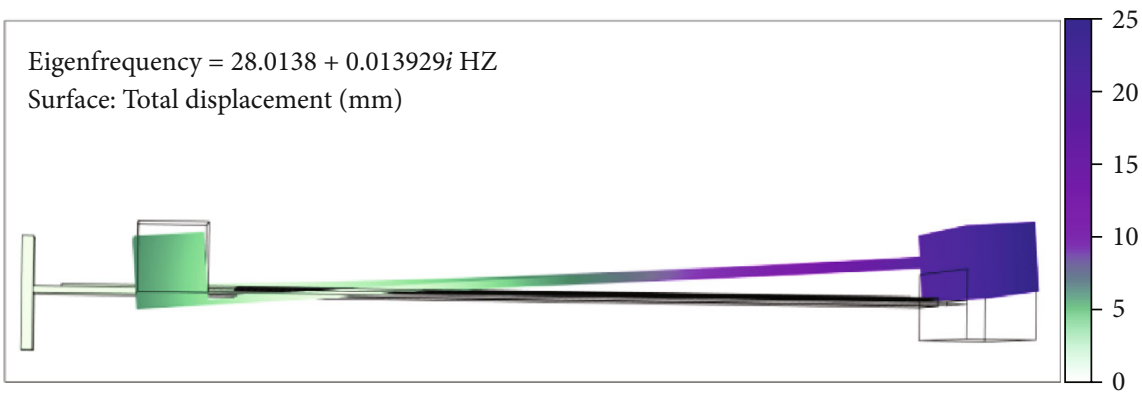

(b)

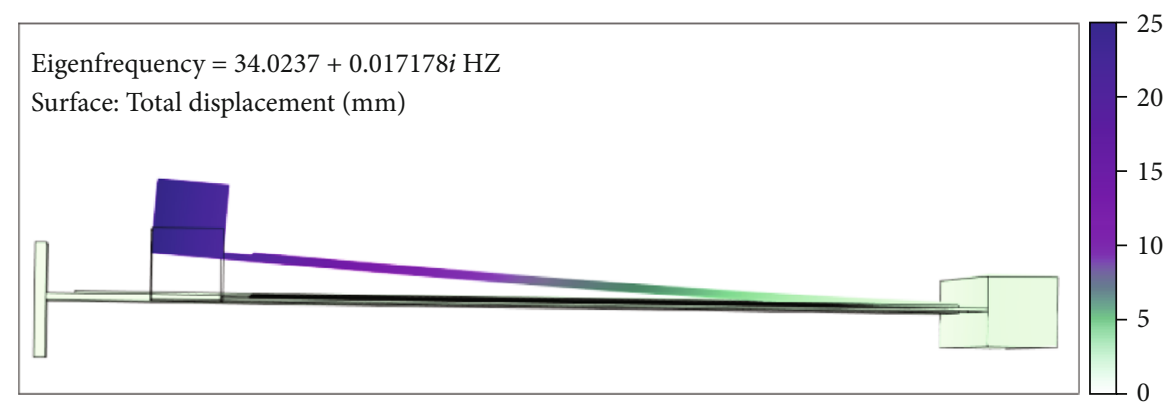

(c)

Figure 6: The simulation results of the first two resonant modes of the C-VEH structure: (a) displacement; (b) the first resonant mode; (c) the second resonant mode.

the $\mathrm{C}-\mathrm{VEH}$ is continuously operating effectively at the frequencies that between the two modes.

\section{Experimental Analysis}

6.1. Experimental Scheme. A prototype device with the design parameters as shown in Table 2 is constructed to evaluate its power generation capability. As shown in Figure 7, upper face of each beam is bonded with two separated piezoelectric sheets. Four piezoelectric sheets connected in parallel and are equipped with electric terminal numbered \#1, \#2, \#3, and \#4, respectively, and another common ground terminal $(\# 0)$. The electric terminals (\#1, \#2,\#3, and \#4) are individually connected to the terminal of load resistors R1, R2, R3, and R4, respectively. The experimental platform (Figure 7) consists of a signal generator (AFG3210C, Tektronix), a power amplifier (YE5871A), a shaker (YE5871), an external resistor box, an accelerometer (Model752A13, Endevco), and a 


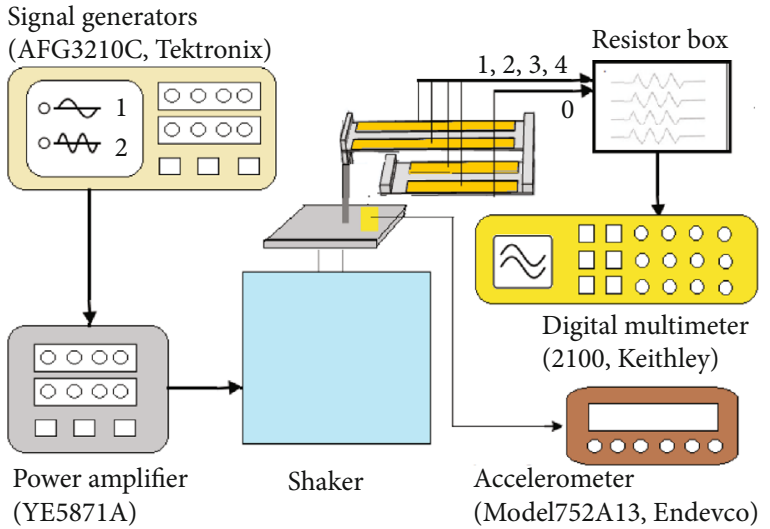

FIgURE 7: Schematic of the experimental setup.

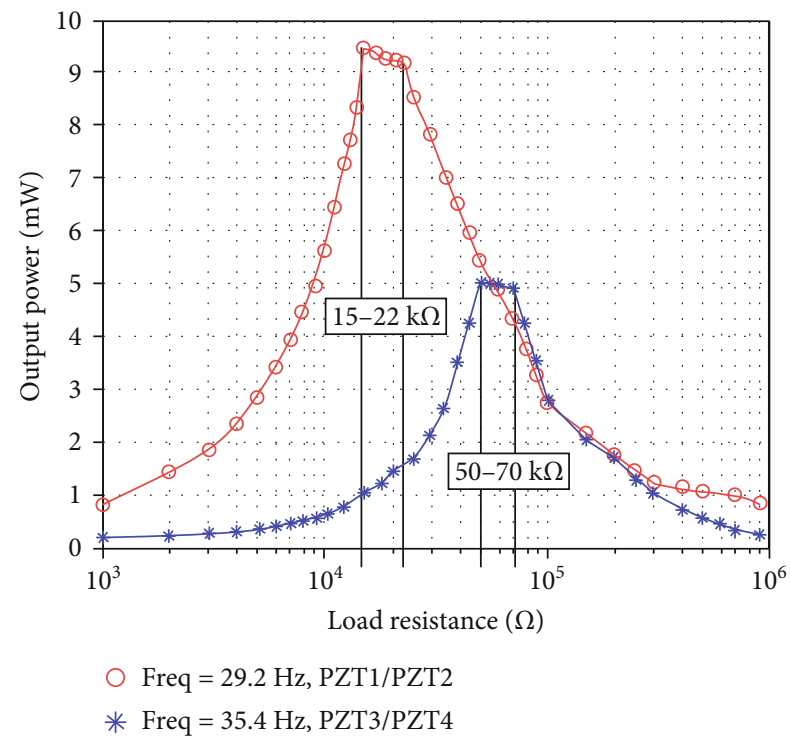

FIgure 8: The output power of the C-VEH versus the load resistance at each resonant frequency, acceleration $=0.7 \mathrm{~g}$.

digital multimeter (2100, Keithley). The C-VEH is assembled on a shaker with a reference accelerometer measuring the acceleration. The signal generator produces the sinusoidal signal that is intensified by a power amplifier to drive the shaker. The root mean square of output voltage $\left(V_{\mathrm{rms}}\right)$ of the $\mathrm{C}-\mathrm{VEH}$ is measured by the digital multimeter. The total output power of the C-VEH can be calculated by the following:

$$
P_{\mathrm{tol}}=\sum_{i}^{n} P_{i}=\sum_{i}^{n} \frac{V_{\mathrm{rms}(i)}^{2}}{R_{i}}
$$

where $P_{\mathrm{tol}}, P_{i}$, and $V_{\mathrm{rms}(i)}$ are the total output power, the output power of the $i^{\text {th }}$ piezoelectric sheet, and the output root mean square voltage of the $i^{\text {th }}$ piezoelectric sheet, respectively.

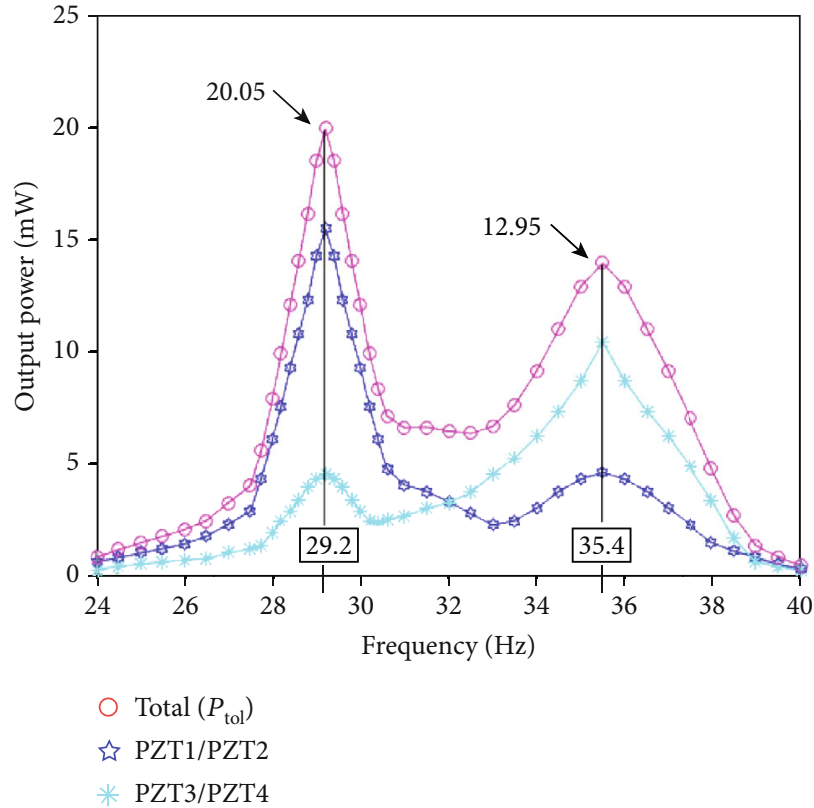

FIgure 9: The output power of the C-VEH versus the excitation frequency at each optimum load resistance, acceleration $=0.7 \mathrm{~g}$.

6.2. Optimal Load Resistance. Experiments are carried out to determine the optimum load resistance of each C-VEH piezoelectric in the case of resonance since the output power of piezoelectric vibration energy harvester is closely related to load resistance. It can be seen from equation (13) that when other parameters are determined, the output power of the piezoelectric vibration energy collector is related to the excitation frequency and load. The reason for the existence of the optimal load is that the piezoelectric structure can be equivalent to a current source with internal resistance. According to the circuit principle, the change of the load has a significant impact on its output power, and there is an optimal load to maximize its output power. According to equation (14), the optimal matching load $\left(R^{\text {opt }}\right)$ of the structure can be calculated theoretically. When measuring the $\left(R^{\text {opt}}\right)$ of the structure, first, sweep the excitation frequency from $10 \mathrm{~Hz}$ to $200 \mathrm{~Hz}$ (step size is $0.2 \mathrm{~Hz}$ ); the maximum output power is obtained when the C-VEH closed-loop resonance frequency is $29.2 \mathrm{~Hz}$ and $35.4 \mathrm{~Hz}$, and the error is less than $5 \%$ compared with the simulation results. Then, the optimal matching load of PZT1/PZT2 is measured. The experimental method is to set the excitation frequency at $29.2 \mathrm{~Hz}$; connect the external load to PZT1/PZT2, and take the measuring points in logarithmic manner within the range of load resistance $1 \times 10^{3} \sim 1 \times 10^{6}$. The optimal matching load of PZT1/PZT2 is roughly between $1 \times 10^{4} \sim 1 \times 10^{5}$ pairs, and in this range, twenty resistance points are measured, and the optimal matching load is in the range of $15 \mathrm{~K} \Omega-22 \mathrm{~K} \Omega$. Finally, the excitation frequency is set at $35.4 \mathrm{~Hz}$, and the optimal matching load of PZT3/PZT4 is in the range of $50 \mathrm{~K} \Omega-70 \mathrm{~K} \Omega$. The experimental results are shown in Figure 8, which exhibit the optimum load resistance range of PZT1/PZT2 and PZT3/PZT4 $(15 \mathrm{~K} \Omega-22 \mathrm{~K} \Omega$ 


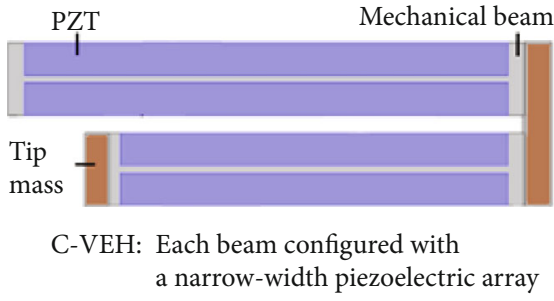

(a)

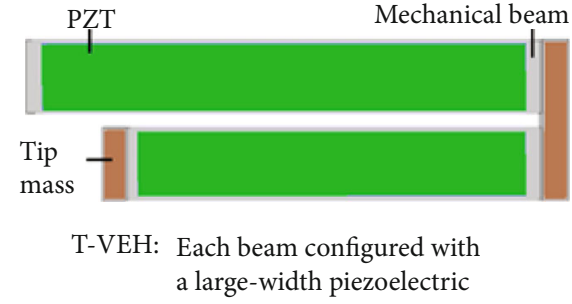

(b)

Figure 10: The C-VEH and T-VEH design.

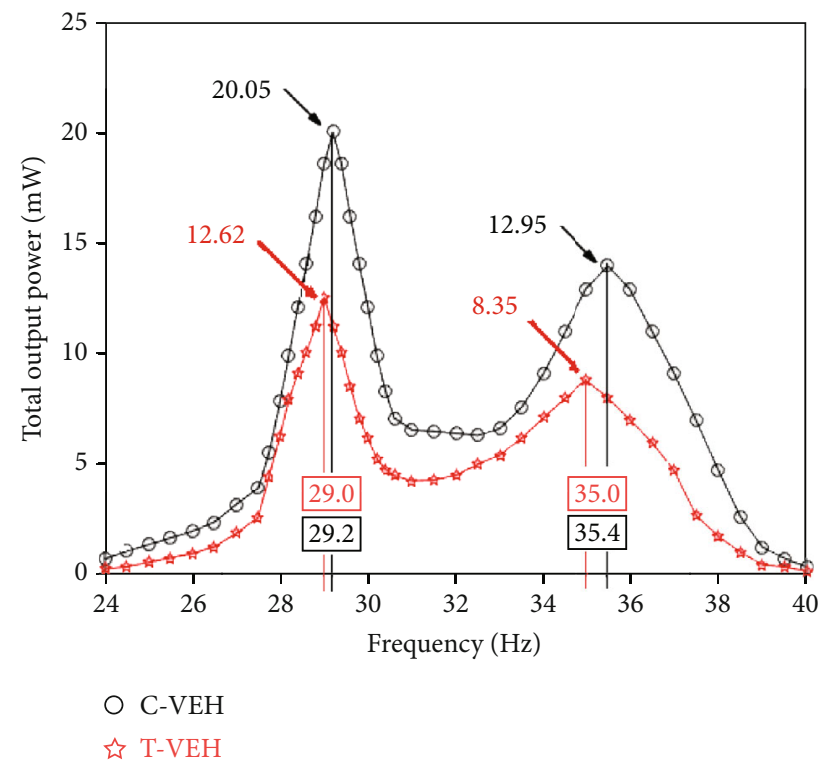

FIGURE 11: The total output power of C-VEH and T-VEH changing with the excitation frequency under their optimal load resistance, acceleration $=0.7 \mathrm{~g}$.

at the first resonant frequency of $29.2 \mathrm{~Hz}$ and $50 \mathrm{~K} \Omega-70 \mathrm{~K} \Omega$ at the second resonant frequency of $35.4 \mathrm{~Hz}$, respectively).

6.3. Output Power. The output power of the prototype is tested with each electric terminal in parallel and connecting to the optimum load resistance. It can be seen from equations (14) and (15) that when other parameters are determined, the output power of the piezoelectric vibration energy harvester is related to the excitation frequency after matching the optimal load, and when the system is in the resonant frequency region, it can obtain larger output power. According to the results of the previous optimal matching load experiment, in the process of the experiment, PZT1/PZT2 is connected with a load of $20 \mathrm{~K} \Omega$, and PZT3/PZT4 is connected with a load of $60 \mathrm{~K} \Omega$. Then, apply excitation signal to the system (the amplitude is $0.5 \mathrm{~mm}$, the frequency scanning range is $20 \mathrm{~Hz}-40 \mathrm{~Hz}$ and the step is $0.5 \mathrm{~Hz}$, and the acceleration is $0.7 \mathrm{~g}$ ) and measure the output power of PZT1/PZT2 and PZT3/PZT4.

The corresponding relationship between the output power and the excitation frequency is shown in Figure 9. The C-VEH achieves two total output power $\left(P_{\text {tol }}\right)$ peaks $(20.5 \mathrm{~mW}$ and $12.95 \mathrm{~mW})$ at frequencies of $29.2 \mathrm{~Hz}$ and
$35.4 \mathrm{~Hz}$. When the excitation frequency is lower than $29.2 \mathrm{~Hz}$ or higher than $35.4 \mathrm{~Hz}, P_{\text {tol }}$ decreases sharply. As the excitation frequency sweeping from $27.5 \mathrm{~Hz}$ to $37.5 \mathrm{~Hz}$, $P_{\text {tol }}$ keeps above $6.48 \mathrm{~mW}$, which is about the half of the peak power outputs. The half-peak-power frequency bands of the designed structure are $27.8-30.6 \mathrm{~Hz}$ and $30.6-37.4 \mathrm{~Hz}$, respectively.

6.4. Discussion. For comparison between the proposed C$\mathrm{VEH}$ of narrow-width piezoelectric arrays (Figure 10(a)) and a traditional large-width piezoelectric structure named T-VEH shown in Figure 10(b), experiment on a T-VEH with the width of piezoelectric sheet on its primary beam is the sum of the widths of the two piezoelectric sheets on $\mathrm{C}-\mathrm{VEH}$ primary beam and the width of the piezoelectric sheet on its secondary beam is the sum of the widths of those on the $\mathrm{C}-\mathrm{VEH}$ secondary beam is conducted. Firstly, the optimal matching resistance of PZT on the primary beam and secondary beams of $\mathrm{T}-\mathrm{VEH}$ is measured, and the results are $8 \mathrm{~K} \Omega-15 \mathrm{~K} \Omega$ and $25 \mathrm{~K} \Omega-30 \mathrm{~K} \Omega$. Then, the primary beam is matched with a load of $10 \mathrm{~K} \Omega$, and the secondary beam is matched with a load of $30 \mathrm{~K} \Omega$. And then, apply excitation signals to the two systems, respectively (the amplitude is $0.5 \mathrm{~mm}$, the frequency scanning range is $20 \mathrm{~Hz}-40 \mathrm{~Hz}$ and the step is $0.5 \mathrm{~Hz}$, and the acceleration is $0.7 \mathrm{~g}$ ), and measure the corresponding situation of the total output power with the excitation frequency. The results are in Figure 11, which shows the comparison of total output powers between C$\mathrm{VEH}$ and T-VEH with respect to the excitation frequency and under their optimal load resistance. For T-VEH, two total output power peaks $(12.62 \mathrm{~mW}$ and $8.35 \mathrm{~mW}$ ) occur at the first two resonant frequencies $(29.0 \mathrm{~Hz}$ and $35.0 \mathrm{~Hz})$, the corresponding optimum load resistance are $10 \mathrm{~K} \Omega$ and $30 \mathrm{~K} \Omega$, respectively, and the half peak power bands are $28.0 \mathrm{~Hz}-30.6 \mathrm{~Hz}$ and $30.8 \mathrm{~Hz}-37.2$, respectively. For C-VEH, two total output power peaks $(20.05 \mathrm{~mW}$ and $12.95 \mathrm{~mW})$ occur at the first two resonant frequencies $(29.2 \mathrm{~Hz}$ and $35.4 \mathrm{~Hz}$ ), the corresponding optimum load resistance are $20 \mathrm{~K} \Omega$ and $60 \mathrm{~K} \Omega$, respectively, and the half peak power bands are $27.8 \mathrm{~Hz}-30.6 \mathrm{~Hz}$ and $30.6 \mathrm{~Hz}-37.4$, respectively. Table 3 shows the comparison of experimental results of the T-VEH and C-VEH. By 1/2-width configuring the piezoelectric sheets, the first two resonant frequencies of both structures almost remain the same, the optimal matching resistance increases, the total output power increases, and the two harmonic response peaks increase by $58.88 \%$ and $55.09 \%$, respectively. That is to say, narrow-width 
TABLE 3: Comparison of experimental results of the C-VEH and the T-VEH.

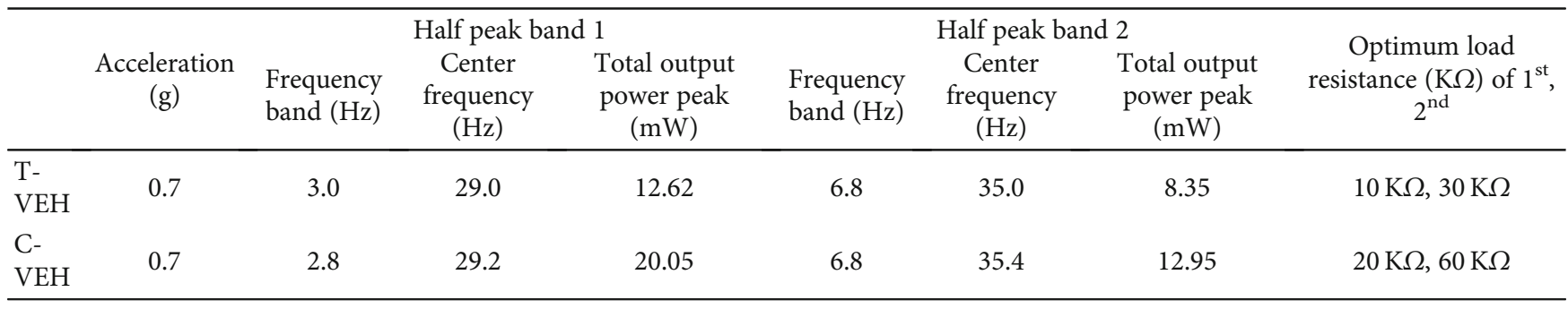

piezoelectric array configuration on the beams displays better power generation capability.

\section{Conclusion}

In summary, a vibration energy harvester based on the structure of two-segment cantilever beam with piezoelectric array configuration for low-frequency vibration energy harvesting has been studied in this letter, which energy conversion efficiency is significantly improved by arraying piezoelectric sheets on cantilever beams, and the operation frequency band is widened by applying two-segment cantilever beams. Our investigation provides a way to improve the energy conversion efficiency and broaden the operation frequency band of piezoelectric vibration energy harvesting structures. The follow-up work is to theoretically discuss and explain why the harvested power by T-VEH is less than C-VEH, that is to say, why energy conversion efficiency is significantly improved by arraying piezoelectric sheets on cantilever beams, and design efficient energy storage circuits.

\section{Data Availability}

There is no data available for this article.

\section{Conflicts of Interest}

The authors declare that they have no conflicts of interest.

\section{Acknowledgments}

This work was supported by the National Natural Science Foundation of China (No. 51965014), the Natural Science Foundation of Guangxi (No. 2018JJA160218), the Aeronautical Science Foundation of China (Grant No. 20180952007), the Foundation of National Key Laboratory on Ship Vibration and Noise (Grant No. 614220400307), and the Foundation of State Key Laboratory of Mechanics and Control of Mechanical Structures (MCMS-I-0520G01).

\section{References}

[1] J. W. Matiko, N. J. Grabham, S. P. Beeby, and M. J. Tudor, "Review of the application of energy harvesting in buildings," Measurement Science and Technology, vol. 25, no. 1, article 012002, 2014.

[2] J. Abdelkefi, M. Scanlon, E. McDowell, and M. R. Hajj, "Performance enhancement of piezoelectric energy harvesters from wake galloping," Applied Physics Letters, vol. 103, no. 3, article $033903,2013$.

[3] L. Tang and J. Wang, "Modeling and analysis of cantilever piezoelectric energy harvester with a new-type dynamic magnifier," Acta Mechanica, vol. 229, no. 11, pp. 4643-4662, 2018.

[4] Z. Xie, C. K. Kwuimy, T. Wang, X. Ding, and W. Huang, "Theoretical analysis of an impact-bistable piezoelectric energy harvester," The European Physical Journal Plus, vol. 134, 2019.

[5] A. Marin, J. Turner, D. S. Ha, and S. Priya, "Broadband electromagnetic vibration energy harvesting system for powering wireless sensor nodes," Smart Materials and Structures, vol. 22, no. 7, article 075008, 2013.

[6] L. J. Gong, Q. S. Pan, W. Li, G. Y. Yan, Y. B. Liu, and Z. H. Feng, "Harvesting vibration energy using two modal vibrations of a folded piezoelectric device," Applied Physics Letters, vol. 107, article 033904, 2015.

[7] C. Eichhorn, F. Goldschmidtboeing, and P. Woias, "Bidirectional frequency tuning of a piezoelectric energy converter based on a cantilever beam," Journal of Micromechanics and Microengineering, vol. 19, no. 9, article 094006, 2009.

[8] W. G. Li, S. Y. He, and S. D. Yu, "Improving power density of a cantilever piezoelectric power harvester through a curved Lshaped proof mass," IEEE Transactions on Industrial Electronics, vol. 57 , no. 3, 2010.

[9] D. Shen, J.-H. Park, J. Ajitsaria, S.-Y. Choe, H. C. Wikle, and D.-J. Kim, "The design, fabrication and evaluation of a MEMS PZT cantilever with an integrated Si proof mass for vibration energy harvesting," Journal of Micromechanics and Microengineering, vol. 18, no. 5, 2008.

[10] D. J. Morris, J. M. Youngsman, and M. J. Anderson, "A resonant frequency tunable, extensional mode piezoelectric vibration harvesting mechanism," Smart Materials and Structures, vol. 17, no. 6, article 065021, 2008.

[11] Q. C. Guan, B. Ju, J. W. Xu, Y. B. Liu, and Z. H. Feng, "Improved strain distribution of cantilever piezoelectric energy harvesting devices using H-shaped proof masses," Journal of Intelligent Material Systems and Structures, vol. 24, pp. 1059-1066, 2013.

[12] D. Huang, S. Zhou, and G. Litak, "Theoretical analysis of multi-stable energy harvesters with high-order stiffness terms," Communications in Nonlinear Science and Numerical Simulation, vol. 69, pp. 270-286, 2019.

[13] T. C. Yuan, J. Yang, and L. Q. Chen, “A harmonic balance approach with alternating frequency/time domain progress for piezoelectric mechanical systems," Mechanical Systems and Signal Processing, vol. 120, pp. 274-289, 2019.

[14] C. Wang, Q. Zhang, and W. Wang, "Low-frequency wideband vibration energy harvesting by using frequency up- conversion 
and quin-stable nonlinearity," Journal of Sound and Vibration, vol. 399, pp. 169-181, 2017.

[15] K. Fan, Q. Tan, H. Liu, Y. Zhang, and M. Cai, "Improved energy harvesting from low-frequency small vibrations through a monostable piezoelectric energy harvester," Mechanical Systems and Signal Processing, vol. 117, pp. 594608, 2019.

[16] S. Nabavi and L. Zhang, "Nonlinear multi-mode wideband piezoelectric MEMS vibration energy harvester," IEEE Sensors Journal, vol. 19, no. 13, pp. 4837-4848, 2019.

[17] Y. J. Wang, T. Y. Chuang, and C. Lee, "Resonant frequency self-tunable piezoelectric cantilevers for energy harvesting and disturbing torque absorbing," Sensors and Actuators A: Physical, vol. 285, pp. 25-34, 2019.

[18] J. Wang, S. Zhou, Z. Zhang, and D. Yurchenko, "High-performance piezoelectric wind energy harvester with Y-shaped attachments," Energy Conversion and Management, vol. 181, pp. 645-652, 2019.

[19] Z. Wu and Q. Xu, "Design and testing of a novel bidirectional energy harvester with single piezoelectric stack," Mechanical Systems and Signal Processing, vol. 122, pp. 139-151, 2019.

[20] Y. Hu and Y. Xu, "A wideband vibration energy harvester based on a folded asymmetric gapped cantilever," Applied Physics Letters, vol. 104, no. 5, article 053902, 2014.

[21] G. Sebald, H. Kuwano, D. Guyomar, B. Ducharne, and S. Mater, "Experimental Duffing oscillator for broadband piezoelectric energy harvesting," Smart materials and structures, vol. 20, no. 10, p. 102001, 2011.

[22] I. H. Kim, H. J. Jung, B. M. Lee, and S. J. Jang, "Broadband energy-harvesting using a two degree-of-freedom vibrating body," Applied Physics Letters, vol. 98, no. 21, p. 214102, 2011.

[23] A. Abdelkefi, F. Najar, A. H. Nayfeh, and S. Ben Ayed, "An energy harvester using piezoelectric cantilever beams undergoing coupled bending-torsion vibrations," Smart Materials and Structures, vol. 20, no. 11, p. 115007, 2011.

[24] A. Hajati and S. G. Kim, "Ultra-wide bandwidth piezoelectric energy harvesting," Applied Physics Letters, vol. 99, no. 8, article 083105, 2011.

[25] M. A. Karami and D. J. Inman, "Parametric study of zigzag microstructure for vibrational energy harvesting," Journal of Microelectromechanical Systems, vol. 21, no. 1, pp. 145-160, 2012.

[26] K. Fan, J. Chang, W. Pedrycz, Z. Liu, and Y. Zhu, “A nonlinear piezoelectric energy harvester for various mechanical motions," Applied Physics Letters, vol. 106, no. 22, p. 223902 , 2015.

[27] H. Hu, L. Dai, H. Chen, S. Jiang, H. Wang, and V. Laude, “Two methods to broaden the bandwidth of a nonlinear piezoelectric bimorph power harvester," Journal of Vibration and Acoustics, vol. 139, no. 3, article 031008, 2017.

[28] X. Rui, Y. Li, Y. Liu, X. Zheng, and Z. Zeng, "Experimental study and parameter optimization of a magnetic coupled piezoelectric energy harvester," Applied Sciences, vol. 8, no. 12, p. 2609, 2018.

[29] B. Zhang, J. Yu, L. Elmaimouni, and X. Zhang, "Magneto-electric effect on guided waves in functionally graded piezoelectric-piezomagnetic fan-shaped cylindrical structures," Materials, vol. 11, no. 11, p. 2174, 2018.

[30] S. Sun and P. W. Tse, "Modeling of a horizontal asymmetric $\mathrm{U}$-shaped vibration-based piezoelectric energy harvester (U-
VPEH)," Mechanical Systems and Signal Processing, vol. 114, pp. 467-485, 2019.

[31] L. R. Alcala-Jimenez, T. P. Jensen, A. Lei, and E. V. Thomsen, "Increased mechanical robustness of piezoelectric magnetoelastic vibrational energy harvesters," Microelectronic Engineering, vol. 207, pp. 19-26, 2019.

[32] V. Apicella, C. S. Clemente, D. Davino, D. Leone, and C. Visone, "Magneto-mechanical optimization and analysis of a magnetostrictive cantilever beam for energy harvesting," Journal of Magnetism and Magnetic Materials, vol. 475, pp. 401-407, 2019.

[33] L. Zhao, S. C. Conlon, and F. Semperlotti, "Broadband energy harvesting using acoustic black hole structural tailoring," Smart Materials and Structures, vol. 23, no. 6, article 065021, 2014.

[34] S. Leadenham and A. Erturk, "Nonlinear M-shaped broadband piezoelectric energy harvester for very low base accelerations: primary and secondary resonances," Smart Materials and Structures, vol. 24, no. 5, article 055021, 2015.

[35] R. L. Harne, A. Sun, and K. W. Wang, "Leveraging nonlinear saturation-based phenomena in an L-shaped vibration energy harvesting system," Journal of Sound and Vibration, vol. 363, pp. 517-531, 2016.

[36] Y. Qin, T. Wei, Y. Zhao, and H. Chen, "Simulation and experiment on bridge-shaped nonlinear piezoelectric vibration energy harvester," Smart Materials and Structures, vol. 28, no. 4, article 045015, 2019.

[37] Y. C. Shu and I. C. Lien, "Analysis of power output for piezoelectric energy harvesting systems," Smart Materials and Structures, vol. 15, p. 1502, 2006. 